\title{
Efficient and facile tuning of Vulcan XC72 with ultra-small Pt nanoparticles for electrocatalytic applications
}

\author{
Adela Eguizabal $^{\mathrm{a}}$, Laura Uson ${ }^{\mathrm{a}}$, Víctor Sebastian ${ }^{\mathrm{a}, \mathrm{b}_{*}, \text { Jose Le }_{\text {. Hueso }}}{ }^{\mathrm{a}, \mathrm{b}}$, and Maria Pilar Pina ${ }^{a, b *}$ \\ Received (in $X X X, X X X)$ XthXXXXXXXXX 20XX, Accepted Xth XXXXXXXXX 20XX \\ ${ }_{5}$ DOI: 10.1039/b000000x
}

In this work, we report the synergic combination of tiny, reproducible, and crystalline platinum nanoparticles with functionalized Vulcan XC72 support for the preparation of efficient electrocatalysts. The morphology and crystallinity of Pt NPs, the amination of carbon support and the Pt loading have been properly evaluated by TEM, STEM, ATR-FTIR, XPS and microwave plasma-atomic emission spectrometry MP-AES. The electrochemical performance of the as prepared powders has 10 been evaluated and compared with the exhibited by commercial Pt-ETEK catalyst with similar Pt-loadings, i.e. $20 \%$ wt. The herein described electrocatalyst shows higher dispersion $\left(98.2 \mathrm{~m}^{2} \mathrm{~g}_{\mathrm{Pt}}{ }^{-1}\right)$ of Pt nanoparticles $(1.5 \pm 0.3 \mathrm{~nm}$ in size) and strong metalsupport interaction than commercial Pt-ETEK counterpart, highlighting the elevated mass activity for methanol electrooxidation.

\section{Introduction}

15 The existing practical catalyst technology for $\mathrm{H}_{2}$ Polymer Electrolyte Membrane (PEM) fuel cells relies on Pt nanoparticles (usually 2-3 nm) supported on high surface area carbon black with metal loadings typically $40-60 \% \mathrm{wt}^{1}$. However, the technical targets of catalyst activity and durability, settled by the U.S DoE ${ }^{2}$ 20 and the European SET-Plan ${ }^{3}$ become more ambitious as the application markets becomes closer. A significant fraction of the cost of a PEM fuel cell comes from precious-metal catalysts that are currently used on the anode and cathode for the electrochemical reactions. Current $R \& D$ focuses on the 25 development of (i) electrocatalysts with reduced precious metal loading, increased activity, improved durability / stability, and increased tolerance to air, fuel and system-derived impurities; and (ii) supports with reduced corrosion, lower cost, and increased non Platinum Group Metal (PGM) catalyst loading. In particular, 30 the 2020 technical target for PGM total loading on electrocatalysts for transportation applications is $0.125 \mathrm{mg}$ $\mathrm{PGM} / \mathrm{cm}^{2}$ electrode area. Thus, alternative catalyst preparation routes have been investigated over the last decade. These can be arranged into four general types: conventional, colloidal, 35 molecular precursor (in liquid or vapour phase) and surface modification. The complexity and lack of reproducibility coming from the high number of experimental variables makes the colloidal synthesis one of the most valuable approaches ${ }^{4}$. This involves the formation of usually prereduced metal nanoparticles, 40 with a narrow particle size distribution, that are then adsorbed or deposited onto the support.

\footnotetext{
${ }^{a}$ Nanoscience Institute of Aragon and Department of Chemical Engineering, University of Zaragoza, C/Mariano Esquillor, s/n, Edif. I+D $+i$, 50018, Zaragoza, Spain.E-mail:victorse@unizar.es mapina@unizar.es; Fax:+34 976 761879; Tel: +34 876555441 ${ }^{b}$ Networking Research Center on Bioengineering, Biomaterials and Nanomedicine, CIBER-BBN, 50018 Zaragoza, Spain
}

Following this approach, the selection of proper platinum precursor, reducing agent and reaction conditions such as 45 concentration, time and temperature are key factors for dispersion and stability control. In our previous work ${ }^{5}$ we have demonstrated the feasibility of using tetrakis-(hydroxymethyl)phosphonium chloride (THPC) as simultaneous reducing and stabilizing agent to obtain Pt nanoparticles under the size of $2 \mathrm{~nm}$ 50 in a single-step synthesis at room temperature. The colloidal suspensions exhibited high stability in aqueous solution for long periods of time, which is highly desirable in electrocatalyst design engineering.

Given the poor stability of supported $\mathrm{Pt}{ }^{6,7}$ to steady state and 55 especially under conditions of load-cycling and high electrode potentials; much recent effort has been devoted to stabilizing Pt nanoparticles. At present, it has been well established that Pt can dissolve under oxidizing potentials $(>0.8 \mathrm{~V})$, re-precipitate and agglomerate due to carbon support corrosion ${ }^{8}$. Functionalization 60 of carbon supports can allow Pt nanoparticles to be firmly immobilized on the surface and retain indispensable properties, such as the dispersion and stabilization of the catalyst. Chen et al. ${ }^{9}$ have described the fabrication of densely loaded, nanosized carbon-supported Pt nanoclusters by conventional impregnation 65 of multichelate-functionalized carbon nanospheres. The existence of ethylenimine chains grafted on the carbon support provides effective steric stabilization through $\mathrm{Pt}-\mathrm{N}$ binding, which prevents the $\mathrm{Pt}$ nanoparticles from aggregating.

Herein, we demonstrate a facile method of attaching ultrafine Pt 70 nanoparticles on the surface of amino- functionalized Vulcan XC72 by simple mixing of the THPC-stabilized Pt colloidal suspension with the carbon powder. The as prepared electrocatalysts exhibit significantly higher electrochemical active surface and catalyst activity toward methanol oxidation than 75 commercial E-TEK. These results indicate that both, the pretreatment of carbon support as well as the facile synthesis and anchoring of tiny $\mathrm{Pt}$ nanoparticles are promising tools to enhance the performance of $\mathrm{Pt}$ based electrocatalysts for PEMFCs 
applications. Furthermore, the proposed strategy can thus be a versatile method for effective immobilization not only of Pt but also other metal colloids over surface modified carbon supports.

\section{Experimental}

\section{${ }_{5}$ Materials}

Commercial Pt catalyst (20 wt\%) was provided by E-TEK, Inc., and Vulcan XC72 carbon used as the supports was obtained from Cabot (USA). All the chemicals reagents, tetrakis (hydroxymethyl) phosphonium chloride solution (80\%wt), 10 sodium borohydride $(99 \% \mathrm{wt})$, poly(vinyl pyrrolidone), hexachloroplatinic acid solution $(8 \% \mathrm{wt})$, sodium hydroxide, nitric acid $(70 \% \mathrm{wt})$, sulfuric acid $(95-98 \% \mathrm{wt})$, and ethylenediamine (75-80\%wt), were of analytical grade, purchased from Sigma Aldrich and used without further purification.

\section{Preparation of Platinum Nanoparticles}

The production of tiny Pt nanoparticles was carried out according to the protocol published in our previous work ${ }^{5}$ and here briefly described. In a typical synthesis of Pt nanoparticles, $100 \mu \mathrm{L}$ of $\mathrm{H}_{2} \mathrm{PtCl}_{6} 8 \%$ wt solution was added to $18 \mathrm{~mL}$ of distilled water in 20 a glass vial under magnetic stirring. Afterwards, $333 \mu \mathrm{L}$ of a 65 $\mathrm{mM}$ THPC solution was added. After several minutes, $165 \mu \mathrm{L}$ of a $1 \mathrm{M} \mathrm{NaOH}$ solution was added to the glass vial. THPC is slowly decomposed by the $-\mathrm{OH}$ anions to formaldehyde and hydrogen, which promotes the reduction of $\mathrm{Pt}$ precursor to 25 metallic nanoparticles. The reaction mixture was kept at room temperature for 4 days, wrapping an aluminium foil to preserve the reaction mixture from photothermal decomposition. The final concentration of the suspension is $0.09 \mathrm{mM}$ in Pt.

\section{Vulcan XC72 functionalization and Pt loading}

30 Vulcan XC72 carbon black conditioning process ${ }^{10}$ was performed according to Scheme 1. Oxygen-containing carbon black is firstly prepared by oxidizing $250 \mathrm{mg}$ of Vulcan XC72 carbon black with $100 \mathrm{~mL}$ of acid solution $\left(\mathrm{H}_{2} \mathrm{SO}_{4}: \mathrm{HNO}_{3}\right.$ ratio $3: 1)$ for $3 \mathrm{~h}$ under vigorous stirring. In a second step, $10 \mathrm{mg}$ of 35 oxidized carbon are mixed with $5 \mathrm{~mL}$ of ethylenediamine (EDA) for $3 \mathrm{~h}$ under sonication. The solid is recovered by centrifugation, thoroughly washed with water and finally dried at $110{ }^{\circ} \mathrm{C}$ in a conventional oven.

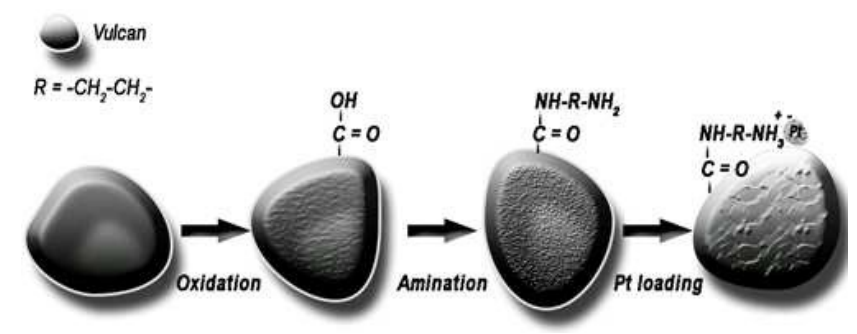

40 Scheme 1 The two-step functionalization process performed on Vulcan XC72 and one-step of Pt loading.

The two-step treatment results in the presence of both $-\mathrm{COOH}$ as well as $-\mathrm{CO}-\mathrm{NH}-\mathrm{R}-\mathrm{NH}_{2}$ on the Vulcan $\mathrm{XC72}$ surface. The "free" amine groups can further attach onto the carbon surface 45 through electrostatic interaction and/or hydrogen-bonding force with the surface carboxyl groups; and eventually, induce aggregation $^{11}$.

Functionalized Vulcan XC72 was added to the Pt colloidal suspension in a ratio of $10 \mathrm{mg}: 3.25 \mathrm{~mL}$, in order to obtain a 50 similar Pt-loading that the commercial electrocatalyst, and left under stirring for 17 hours on a rotating roller mixer. The resulting product, denoted as Pt-Vulcan, was centrifuged and washed with DDI water and dried in a vacuum oven. The presence of functional groups on Vulcan XC72 enhances the Pt ${ }_{55}$ dispersion and stability thanks to the interactions between the electron-rich-nitrogen present in the amino groups of the Vulcan $\mathrm{XC72}$, and the Pt orbitals via their lone pairs ${ }^{11}$. Indeed, the $\mathrm{Z}$ potential is $7.04 \mathrm{mV}$ for functionalized Vulcan XC72 and -30.57 $\mathrm{mV}$ for Pt NPs.

\section{${ }_{60}$ Instrumentation and measurements}

ATR-FTIR (VERTEX 70 equipment with microscope slide MKII Colden Gate ATR from 4000 to $600 \mathrm{~cm}^{-1}, 256$ scans and resolution of $0.05 \mathrm{~cm}^{-1}$ ) and XPS analysis (Axis Ultra DLD (Kratos Tech.) equipment and exciting by the monochromatized ${ }_{65} \mathrm{AlK} \alpha$ source $(1486.6 \mathrm{eV})$ at $15 \mathrm{kV}$ and $10 \mathrm{~mA}$ ) were performed to check the functionalization of Vulcan XC72 with amine groups and evaluate the oxidation states of the Pt nanoparticles. All the Binding Energies were internally standardized to the graphitic $\mathrm{C} 1 \mathrm{~s}$ peak at $285 \mathrm{eV}$ and the background was subtracted by the 70 Shirley method.

The morphology and the size of the Pt nanoparticles and Pt based electrocatalysts were examined by TEM. Samples for TEM were prepared by drop-casting $5 \mu \mathrm{L}$ of the colloidal suspension onto a carbon-coated copper grid after ultrasonic dispersion in ethanol 75 and allowed to dry in air before analysis. Preliminary electron microscopy observations were carried out using a T20-FEI microscope with a $\mathrm{LaB}_{6}$ electron source fitted with a "SuperTwin1", objective lens allowing a point to point resolution of $2.4 \AA$. Aberration corrected scanning transmission electron 80 microscopy images were acquired using a high angle annular dark field detector in a FEI XFEG TITAN electron microscope operated at $300 \mathrm{kV}$ equipped with a CETCOR Cs probe corrector from CEOS Company allowing forming an electron probe of 0.08 $\mathrm{nm}$. The geometric aberrations of the probe-forming system were 85 controlled to allow a beam convergence of $24.7 \mathrm{mrad}$ half-angle to be selected. Elemental analysis was carried out with EDS (EDAX) detector which allows performing EDX experiments in scanning mode.

The Pt loading was determined by MP-AES (Agilent 90 Technologies 4100). Electrocatalyst samples were digested by aqua regia in microwave oven (millestone ethos plus) at $200{ }^{\circ} \mathrm{C}$ for $15 \mathrm{~min}$.

\section{Electrochemical characterization}

The electrochemical experiments were performed using an 95 Autolab PGSTAT 101 potentiostat controlled by a dedicated software packages NOVA 1.8 in a standard three-electrode Pyrex cell. The procedure adapted is similar to the methods reported in literature ${ }^{12}$. A graphite rod and a reversible hydrogen electrode (RHE) were used as counter and reference electrodes, 100 respectively. A thin layer of the electrocatalyst was deposited onto a glassy carbon disk ( $7 \mathrm{~mm}$ in diameter) working electrode. A suspension of the electrocatalyst was prepared by ultrasonication of Pt based catalysts in $15 \mu \mathrm{l}$ of Nafion (Aldrich, 
$15 \%$ wt in water) and $500 \mu \mathrm{L}$ MiliQ water. An aliquot of $40 \mu \mathrm{L}$ of suspension was deposited on top of the graphite disk and dried. Dilute liquid electrolytes, $0.5 \mathrm{M} \mathrm{H}_{2} \mathrm{SO}_{4}$, were degassed by purging $\mathrm{N}_{2}$ for $3 \mathrm{~h}$ prior to every measurement. Several cycles at 5 high scan rate $\left(500 \mathrm{mVs}^{-1}\right)$ were also carried out before the electrochemical measurements to remove any residual THPC or by-products and clean the Pt surface.

Electro-oxidation of $\mathrm{CO}$ was performed to (i) confirm the presence of electroactive $\mathrm{Pt}$ sites and (ii) estimate the 10 electrochemical active surface (EAS) area of the catalysts. Cyclic voltammograms were recorded between $0.01 \mathrm{~V}$ and $1.20 \mathrm{~V}$ vs. RHE at a scan rate of $20 \mathrm{mVs}^{-1}$ and room temperature. CO stripping was conducted after bubbling this gas in the cell for 10 $\min$ at $0.20 \mathrm{~V}$ (the potential value where maximum CO coverage

15 on $\mathrm{Pt}^{12}$ is achieved), followed by nitrogen purging to remove the excess of $\mathrm{CO}$. A factor of $420 \mu \mathrm{C} \cdot \mathrm{cm}^{-2}$ has been considered for conversion of charge to surface area of platinum ${ }^{12}$.

The cyclic voltammetry (CV) experiments were also performed in a $0.5 \mathrm{M} \mathrm{H}_{2} \mathrm{SO}_{4}$ solution in the absence and presence of $2 \mathrm{M}$

${ }_{20} \mathrm{CH}_{3} \mathrm{OH}$ between $0.05 \mathrm{~V}$ and $1.20 \mathrm{~V}$ vs. RHE at $35 \mathrm{mVs}^{-1}$. Stable voltammograms recorded after at least 20 cycles were taken into account for data interpretation. Chronoamperometry experiments were carried out in $0.5 \mathrm{M} \mathrm{H}_{2} \mathrm{SO}_{4}$ solution as electrolyte with $\mathrm{CH}_{3} \mathrm{OH} 2 \mathrm{M}$ at $0.6 \mathrm{~V}$ vs. RHE for $600 \mathrm{~s}$.

\section{${ }_{25}$ Results and discussion}

\section{Carbon Functionalization and Pt anchoring}

Figure 1 shows the FT-IR analysis carried out in order to confirm the amine-functionalization of the carbon Vulcan support. Typical bands at 1505 and $1650 \mathrm{~cm}^{-1}$ attributed to bending 30 deformation of $\mathrm{N}-\mathrm{H}$ bonds ${ }^{11}$ are indicative of the successful presence of free amine terminal groups onto the surface Vulcan XC72 after functionalization, whereas no characteristic bands appear in FTIR spectra of pristine support.

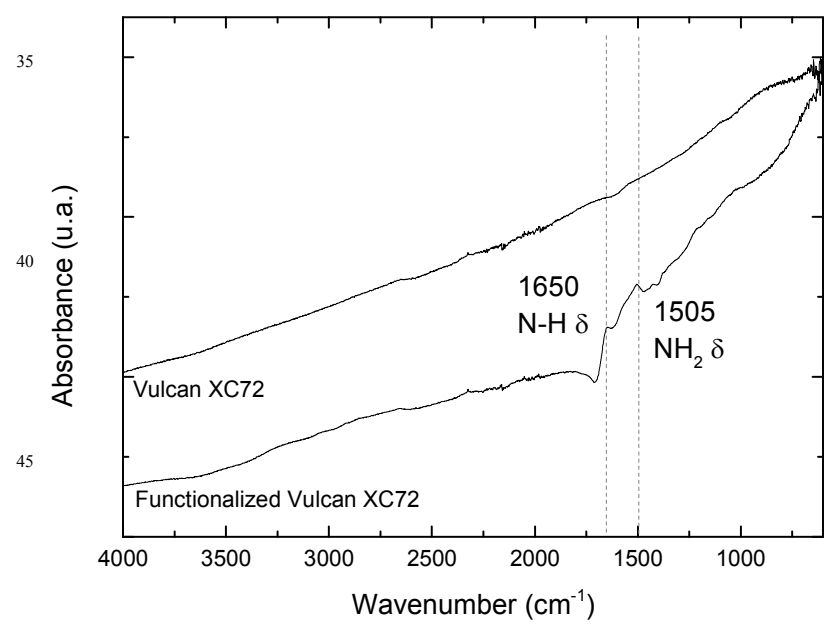

Figure 1 FTIR spectra of pristine Vulcan XC72 and functionalized ${ }_{50}$ Vulcan XC72 with amine groups

In addition, the XPS analyses of Vulcan XC72 after functionalization, shown in Figure 2, further corroborate the presence of nitrogen (up to 10 atomic \%). The N1s spectrum can be explained by the major contribution of primary amine terminal
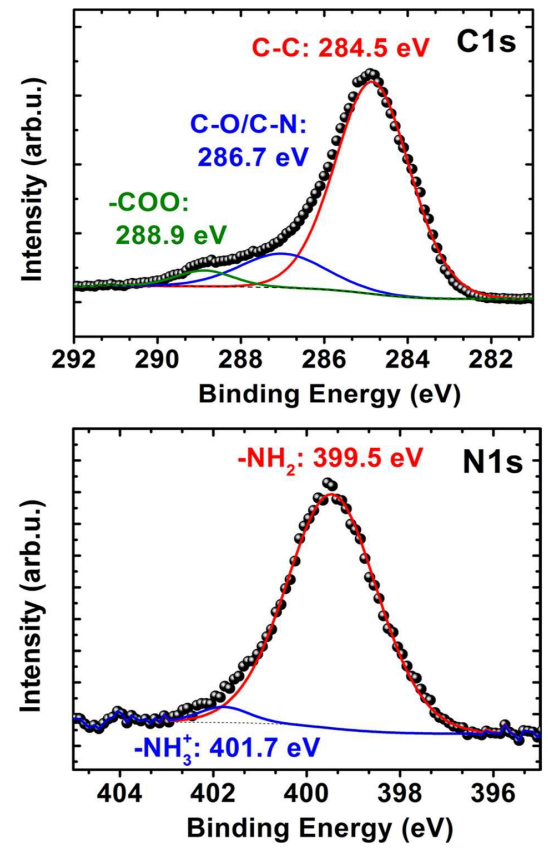

Figure $2 \mathrm{X}$-ray photoemission spectra and curve fittings corresponding to the C1s (top) and N1s areas (bottom) of Vulcan XC72 after 75 functionalization with EDA.

groups corresponding to BEs of $399.5 \mathrm{eV}^{13}$ and a minimal contribution at $401.7 \mathrm{eV}$ previously reported for EDA ${ }^{14}$ and tentatively assigned to protonated amine groups $\left(-\mathrm{NH}_{3}{ }^{+}\right)^{15}$. Furthermore, the $\mathrm{C} 1 \mathrm{~s}$ spectrum also confirms the 80 functionalization of carbon with $\mathrm{C}-\mathrm{O}$ and $\mathrm{C}-\mathrm{N}$ species at 286.7 $\mathrm{eV}$ and an excess of carboxylic groups at $288.9 \mathrm{eV}$ from the preliminary oxidation step that did not react with EDA to render the amide bonding (Figure 2).

The TEM micrographs for Pt nanoparticles obtained in the 85 presence of THPC are shown in Figure 3 a-b. TEM analysis revealed that both the soft reduction of Pt precursor and the stabilization achieved by THPC led to well-crystallized structures which could be indexed according to the Fm-3m space group. The high-resolution STEM image shows the lattice fringe 90 distance of $0.223 \mathrm{~nm}$ corresponding to $\mathrm{Pt}\left(\begin{array}{lll}1 & 1 & 1\end{array}\right)$, which demonstrates the highly crystalline character of metallic $\mathrm{Pt}$ nanoparticles. The size distribution study reveals that tiny monodisperse NPs with a size of $1.5 \pm 0.3 \mathrm{~nm}$ were achieved. Figure $3 \mathrm{~b}$ and its inset reveal the single crystal structure of $\mathrm{Pt}$ ${ }_{95}$ NPs. The hydrosols obtained presented an excellent colloidal stability, without precipitation or agglomeration over a period of several months. These facts highlight that THPC is a key molecule to produce nanoparticles under a narrow size distribution and time-stability, which is highly desirable to load 100 the carbon support and form the electrocatalyst.

Figure 3-c-d shows a typical STEM image of the Pt-Vulcan catalyst after anchoring the Pt NPs. It can be observed the globular morphology of Vulcan XC72, with an average diameter of approx. $80 \mathrm{~nm}$. 

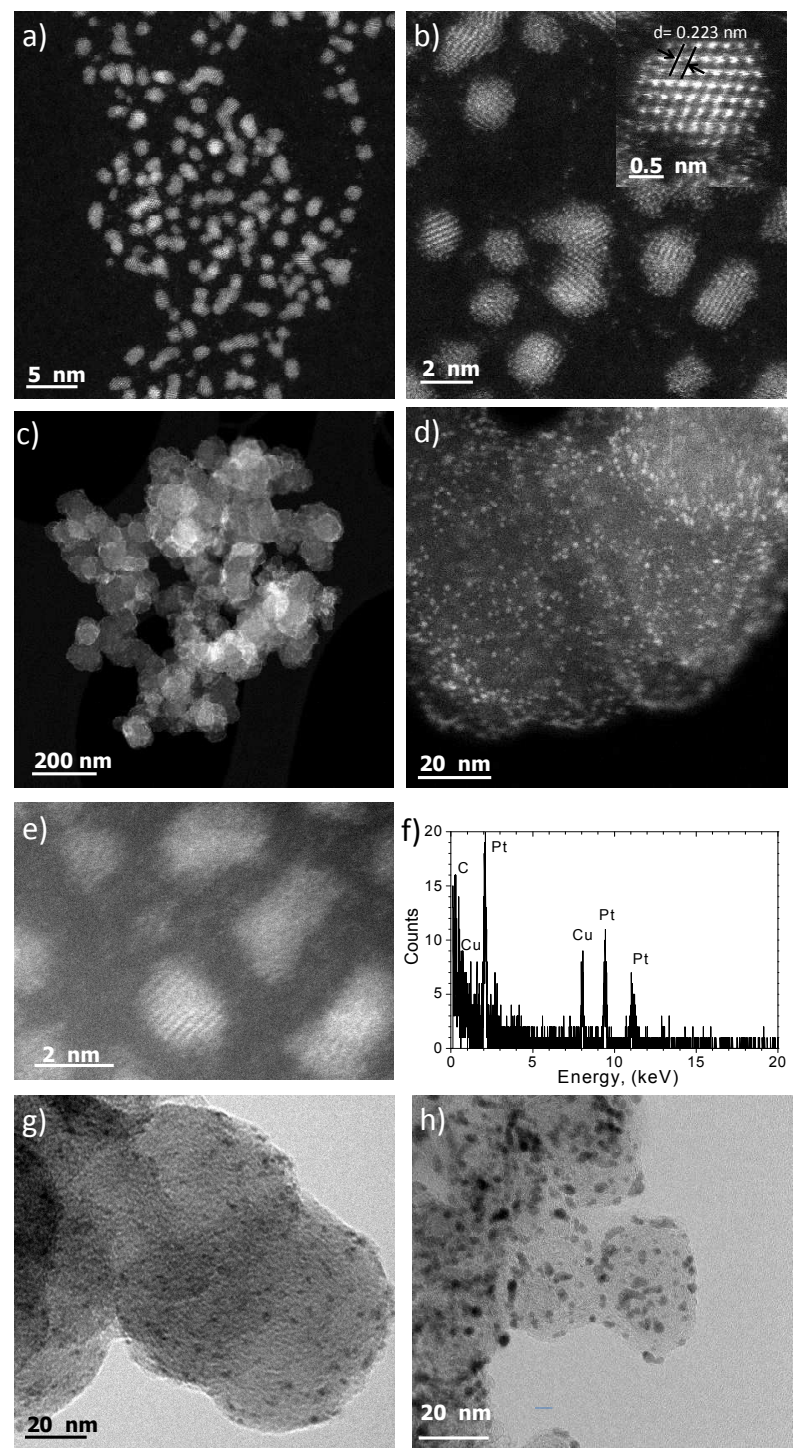

Figure 3 STEM-HAADF Micrographs. a) As-made Pt nanoparticles. b) Atomic resolution image from Pt nanoparticles. Inset is a magnification of a Pt nanoparticle showing a single crystal structure. c) Pt-Vulcan. d) 5 Homogenous distribution of Pt nanoparticles on the modified surface of Vulcan XC72 support. e) Detail image with atomic resolution of Pt nanoparticles anchored to the amino functionalized Vulcan XC72. f) EDX spectrum analysis of Pt nanoparticles at the functionalized Vulcan XC72 surface. TEM micrographs: g) Pt-Vulcan, h) Pt-ETEK (20 \%wt Pt).

10 The high magnification image reported in Figure 3-d shows that Pt nanoparticles are highly dispersed on the surface of the Vulcan $\mathrm{XC72}$. The effectiveness of both grafting and anchoring processes were successfully achieved since it was verified that $\mathrm{Pt}$ NPs were only located at the surface of Vulcan XC72 NPs and 15 not at the grid carbon film. Figure 3-e shows a high resolution image of immobilized Pt nanoparticles on the Vulcan XC72 nanospheres. The EDX spectrum analysis confirms the presence of Pt nanoparticles at the functionalized Vulcan XC72 surface (Figure 3-f). Figure 3-g, h depicts TEM micrographs of both Pt20 Vulcan and the commercial Pt-ETEK electrocatalysts. Pt-Vulcan electrocatalyst contains a similar Pt loading, with a size under 2 $\mathrm{nm}$. On the other hand, Pt-ETEK electrocatalyst is densily loaded with Pt nanocrystallites whose size distribution ranges from 1.5 $\mathrm{nm}$ to $6 \mathrm{~nm}$. Consequently, it is expected that the high dispersion
25 achieved of tiny Pt nanoparticles supported on Vulcan XC72 nanospheres would result in higher electrochemical surface area and enhanced catalytic performance.

XPS analysis of the Pt4f region has been employed to obtain additional information about the chemical oxidation state at the 30 surface of the Pt NPs as well as to evaluate the interaction between the noble metal particles and the carbon supports. Figure 4 shows the Pt4f electron spectra of the commercial Pt-ETEK (Figure 4-top) and the functionalized Vulcan support after the assembly of the Pt nanoparticles (Figure 4-bottom), respectively. 35 The commercial catalyst exhibits three doublets accounting for the spin-orbital splitting of the $4 f_{7 / 2}$ and $4 f_{5 / 2}$ states and the different oxidation states of the Pt nanoparticles. The lower core binding energies for $\mathrm{Pt}_{4 / 2}$ at $72 \mathrm{eV}$ are attributed to $\mathrm{Pt}$ in its metallic state $(\mathrm{Pt}-0)^{16}$. Its contribution is significantly higher than 40 the species associated to $\mathrm{Pt}$ in its oxidized states as $\mathrm{Pt}^{2+}$ : $\mathrm{PtO} / \mathrm{Pt}(\mathrm{OH})_{2}$ or in a minor extent as $\mathrm{Pt}^{4+}: \mathrm{PtO}_{2} / \mathrm{Pt}(\mathrm{OH})_{4}$. These latter species are detected at higher core binding energies centered at $73 \mathrm{eV}$ and $74 \mathrm{eV}$ for $\mathrm{Pt}_{4} \mathrm{f}_{7 / 2}$, respectively ${ }^{16}$.

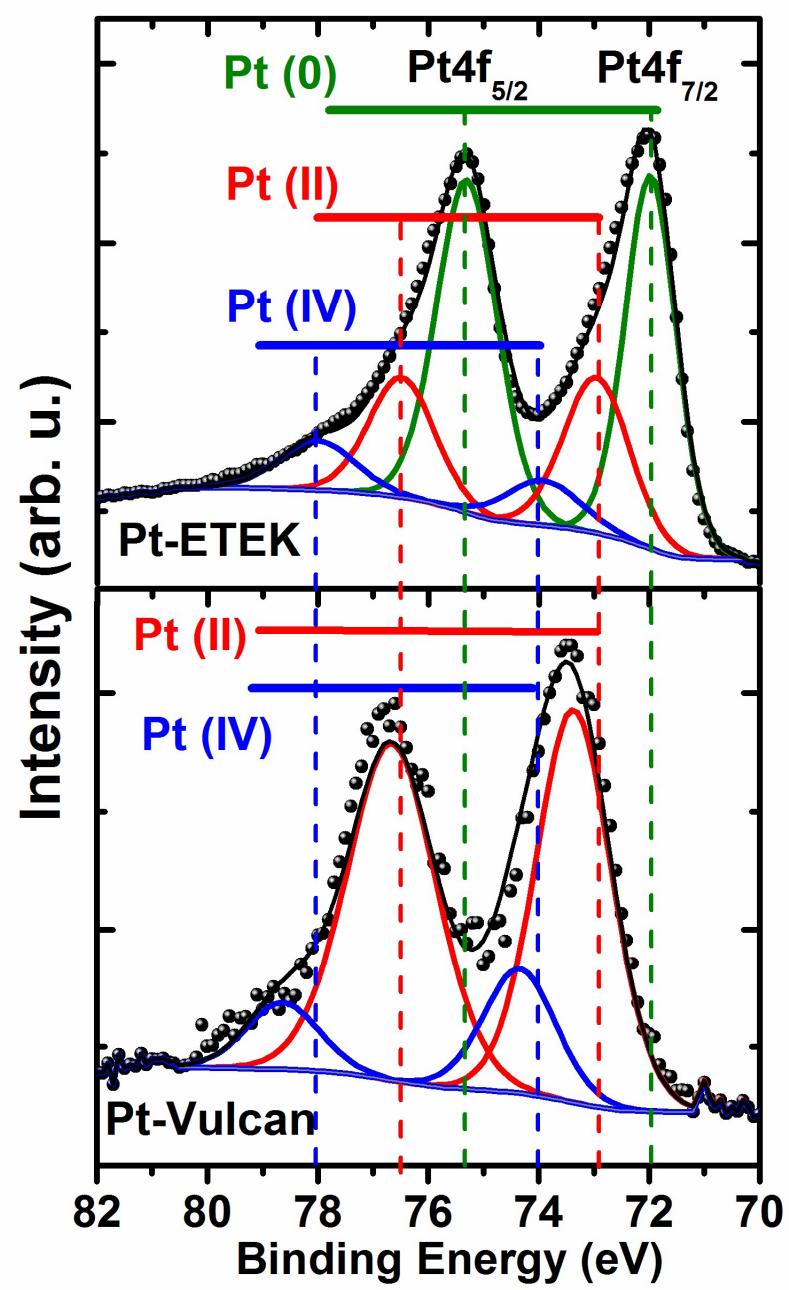

Figure 4 X-ray Photoelectron spectra of Pt4f levels corresponding to: 45 (top) Pt-ETEK and (bottom) Pt-Vulcan samples, respectively.

The XPS spectrum corresponding to the Pt-Vulcan sample only displays two doublets attributed to the Pt-II and Pt-IV oxidized species (Figure 4-bottom). Interestingly, the binding energies are 
slightly shifted towards higher energies $(73.6 \mathrm{eV}$ and $74.5 \mathrm{eV}$ respectively for $\mathrm{Pt}_{4} \mathrm{f}_{7 / 2}$ ). Previous works have associated this positive shift to a decrease in the electronic charge density on the Pt atoms due to electron transfer from the metal to the carbon 5 support ${ }^{16,17,18}$. In our particular case, this effect is favoured by the presence of amine groups on the Vulcan support ${ }^{18}$. The absence of metallic species also accounts for: (i) the high dispersion and accessibility; and, (ii) the tiny size of Pt NPs that are more prone to oxidation upon ambient air exposure ${ }^{19}$.

\section{${ }_{10}$ Electrochemical characterization of the catalyst}

Figure 5-a shows the cyclic voltammogram in $0.5 \mathrm{M} \mathrm{H}_{2} \mathrm{SO}_{4}$ for the Pt-Vulcan catalyst. The dual sorption/desorption peaks in the negative region can be attributed to hydrogen redox activity on $\mathrm{Pt}$ nanoparticles (see Figure 5-b). This CV response corresponds 15 with a typical polycrystalline Pt activated ${ }^{20}$. The medium potential region represents the double layer. The characteristic profile in the positive region can be attributed to the oxygen like species $(\mathrm{Pt}-\mathrm{O}, \mathrm{Pt}-\mathrm{OH})$. The hydrogen adsorption/desorption waves are clearly observed between 0.01 and $0.15 \mathrm{~V}$ (see Figure 20 -b).
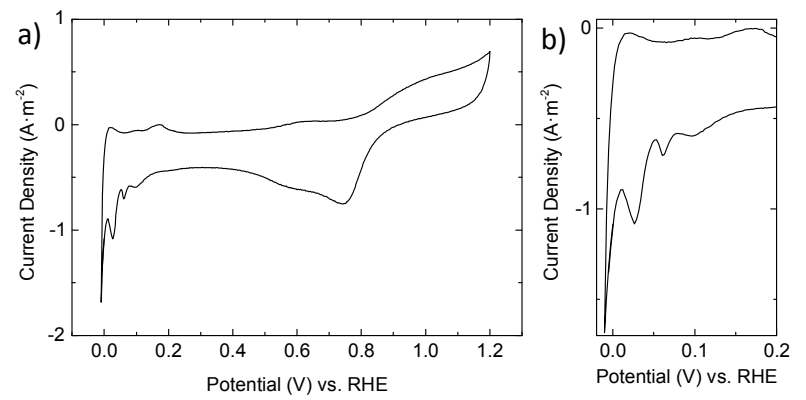

Figure 5 a) Cyclic voltammogram $\left(20 \mathrm{mVs}^{-1}\right)$ for the Pt-Vulcan catalyst in $0.5 \mathrm{M} \mathrm{H}_{2} \mathrm{SO}_{4}$. b) A detail of the voltammogram at the $\mathrm{H}_{2}$ adsorption/desorption region.

${ }_{25}$ Figure 6 compares the cyclic voltammetry measurements for both Pt-Vulcan and the commercial Pt-ETEK electrocatalysts obtained during the stripping of a $\mathrm{CO}$ monolayer formed at $0.20 \mathrm{~V}$, as well as the second cycle after $\mathrm{CO}$ oxidation, which corresponds to the voltammogram in the base electrolyte $\left(0.5 \mathrm{M} \mathrm{H}_{2} \mathrm{SO}_{4}\right)$ for the 30 clean $\mathrm{Pt}$ surface. The peak between $0.7 \mathrm{~V}$ and $0.9 \mathrm{~V}$ in the forward scans corresponds to the adsorbed $\mathrm{CO}$ oxidation.

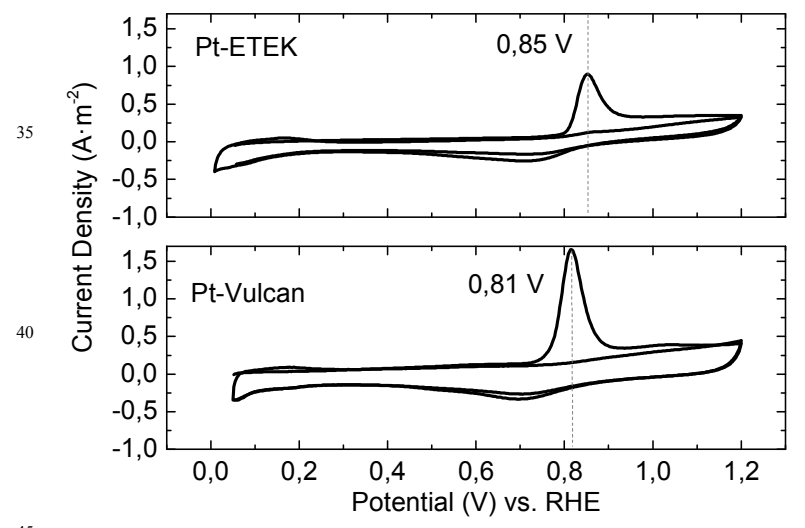

Figure 6 Cyclic voltammograms $\left(20 \mathrm{mVs}^{-1}\right)$ of $\mathrm{CO}$ stripping for the $\mathrm{Pt}$ based electrocatalysts studied in this work.
Moreover, the relative position of the peaks indicates that the electrocatalyst herein prepared is less susceptible to be poisoned so by $\mathrm{CO}$ presence than commercial counterpart, whose maximum potentials are $0.81 \mathrm{~V}$ and $0.85 \mathrm{~V}$, respectively.

Table 2 shows the EAS area values calculated from the coulombic amount associated with the $\mathrm{CO}$ oxidation peak area. It is noted that commercial Pt-ETEK shows a surface area of 3.86 ${ }_{55} \mathrm{~cm}^{2}$ while Pt-Vulcan shows $3.98 \mathrm{~cm}^{2}$. Once expressed the EAS values per $\mathrm{Pt}$ loading on the electrode, it is noted that $\mathrm{Pt}$ nanoparticles deposits over the amino functionalized Vulcan XC72 gives higher Pt dispersion, i.e. Pt utilization, than commercial counterpart. Thus, the experimental value of $\mathrm{Pt}$ 60 dispersion for Pt-Vulcan is $98.2 \mathrm{~m}^{2} \mathrm{~g}_{\mathrm{Pt}}{ }^{-1}$ vs. $82.4 \mathrm{~m}^{2} \mathrm{~g}_{\mathrm{Pt}}{ }^{-1}$ estimated for commercial Pt-ETEK.

Table 2 Some characteristics of the electrocatalysts herein studied

\begin{tabular}{|c|c|c|c|}
\hline Sample & $\begin{array}{c}\text { Pt content (\%) } \\
(\text { MP-AES) }\end{array}$ & $\begin{array}{c}\text { EAS } \\
\left(\mathrm{cm}^{2}\right)\end{array}$ & $\begin{array}{c}\text { Pt dispersion } \\
\left(\mathrm{m}^{2} \mathrm{~g}_{\mathrm{Pt}}{ }^{-1}\right)\end{array}$ \\
\hline Pt-Vulcan & 19.4 & 3.93 & 98.2 \\
\hline Pt-ETEK & 20 & 3.86 & 82.4 \\
\hline
\end{tabular}

${ }_{65}$ The electrocatalytic activities of the Pt based catalysts were examined in $0.5 \mathrm{M} \mathrm{H}_{2} \mathrm{SO}_{4}$ solution containing $2 \mathrm{M} \mathrm{CH}_{3} \mathrm{OH}$ at a scan rate of $35 \mathrm{mV} \mathrm{s}^{-1}$. The currents were normalized by the mass of Pt material for comparison (see Figure 7). The voltammetric features are in good agreement with literature ${ }^{21,22}$.

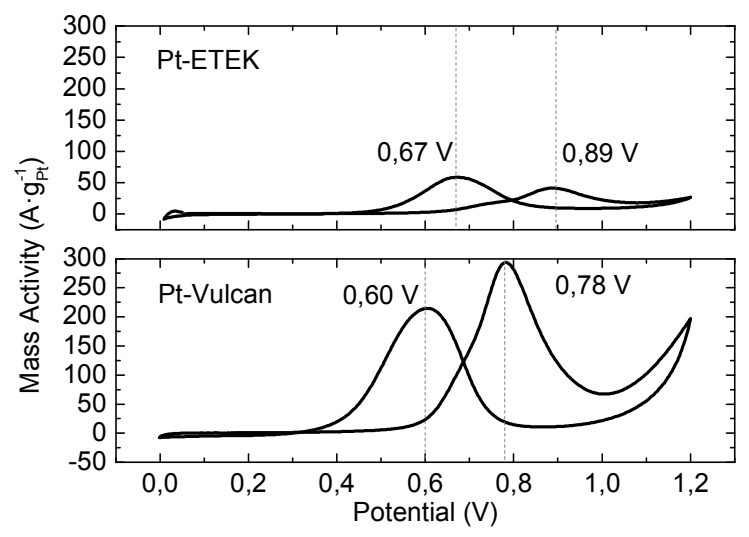

70

Figure 7 Cyclic voltammograms $\left(35 \mathrm{mVs}^{-1}\right)$ for Pt based elecctrocatalysts in $2 \mathrm{M} \mathrm{CH}_{3} \mathrm{OH}+0.5 \mathrm{M} \mathrm{H}_{2} \mathrm{SO}_{4}$

The oxidation current increases for the Pt based electrocatalysts until the potential reaches a maximum above 0.8 vs. RHE and 75 starts to decrease because the formation of Pt oxides inhibits methanol oxidation at this higher potential range. It is clearly noticeable that the oxidation current with Pt-Vulcan is considerably higher than that of Pt-ETEK catalyst. This significant improvement in the catalytic performance can be 80 attributed to the high level of dispersion of Pt nanoparticles with a narrow size distribution over the amino modified Vulcan XC72, and the stronger electron interactions between the $\mathrm{Pt}$ nanoparticles and the support as revealed by XPS of Pt $4 \mathrm{f}$ core level (see Figure 4).

${ }_{85}$ The anodic peak current density in the forward scan increases 
from 43 for Pt-ETEK to $290 \mathrm{Ag}_{\mathrm{Pt}}{ }^{-1}$ for Pt-Vulcan. Under identical experimental conditions ( $2 \mathrm{M} \mathrm{CH}_{3} \mathrm{OH}+0.5 \mathrm{M} \mathrm{H}_{2} \mathrm{SO}_{4}$ ), mass specific activity values for methanol electrooxidation over $\mathrm{Pt}$ nanoparticles loaded on Vulcan XC72 (EAS=48 $\left.\mathrm{m}^{2} \mathrm{~g}_{\mathrm{Pt}}{ }^{-1}\right)$ of 26.4 ${ }_{5} \mathrm{Ag}_{\mathrm{Pt}}{ }^{-1}$ has been reported in the literature ${ }^{23}$.

Moreover, there is a shift in the potential at which the peak current arises: $0.78 \mathrm{~V}$ for the Pt-Vulcan and $0.89 \mathrm{~V}$ for Pt-ETEK. This shift could result from differences in the distribution of exposed Pt crystal faces exposed on the tested sample. 10 Additionally, the current density ratio of the forward oxidation peak $(0.78 \mathrm{~V})$ to the backward oxidation peak $(0.60 \mathrm{~V})$, is usually used to describe the anti-poisoning capability of catalysts. The larger this value, the more tolerant the catalyst is towards intermediate carbonaceous species. As it can be observed in ${ }_{15}$ Figure 7, the value of Pt-Vulcan is larger than Pt-ETEK, indicating that poisoning intermediates are easily removed from the Pt electrocatalyst herein developed.

In order to evaluate the performance with operation time, experiments of chronoamperometry have been carried out for 20 both electrocatalysts (Figure 8). The applied potential is fixed in $0.6 \mathrm{~V}$ for $600 \mathrm{~s}$. Pt-Vulcan electrocatalyst presents a drop slower than Pt-ETEK catalyst. This fact indicates again that the catalyst based on tiny Pt NPs and aminated carbon support performs the better than the commercial counterpart. The mass activity for Pt${ }_{25}$ ETEK is $10.4 \mathrm{Ag}_{\mathrm{Pt}}{ }^{-1}$ to $600 \mathrm{~s}$, whereas that, it is $24.5 \mathrm{Ag}_{\mathrm{Pt}}{ }^{-1}$ for PtVulcan, adequate value for the purpose of this work.

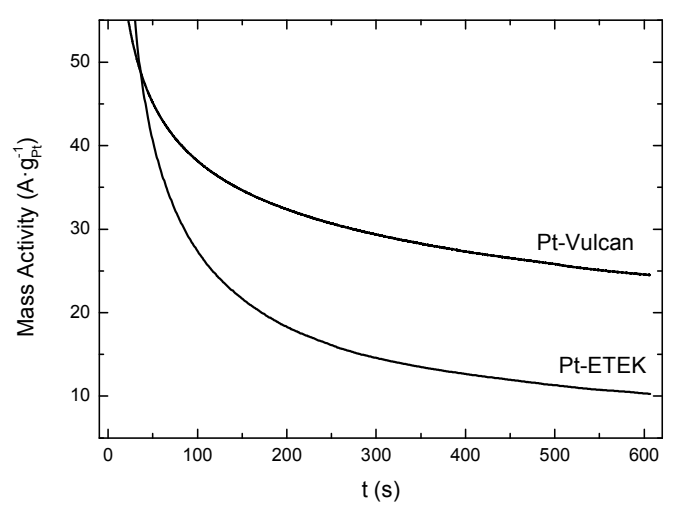

Figure 8 Chronoamperometry plot for the electrooxidation of $\mathrm{CH}_{3} \mathrm{OH} 2$ $\mathrm{M}$ in $\mathrm{H}_{2} \mathrm{SO}_{4} 0.5 \mathrm{M}$ at $0.6 \mathrm{~V}$ vs. RHE on the electrocatalysts based on Pt.

\section{${ }_{30}$ Conclusions}

A facile and straightforward approach for the preparation of electroactive catalysts for methanol oxidation based on the anchoring of crystalline, ultrafine Pt nanoparticles on the surface of amino- functionalized Vulcan XC72 has been developed. Due 35 to the presence of well dispersed Pt nanoparticles (below $2 \mathrm{~nm}$ in size) on the surface of Vulcan, the exhibited electrochemical active surface is larger than the evaluated for Pt-ETEK counterpart (98.2 vs, $82.4 \mathrm{~m}^{2} \mathrm{~g}_{\mathrm{Pt}}{ }^{-1}$, respectively). Pt-Vulcan presented the highest current densities towards methanol 40 electrooxidation, about six times higher than Pt-ETEK counterpart (290 vs. $43 \mathrm{Ag}_{\mathrm{Pt}}{ }^{-1}$ respectively). This electrocatalytic outperformance is attributed to the higher dispersion and utilization of the Pt particles and the stronger metal-support interactions. Furthermore, the tolerance towards $\mathrm{CO}$ and 45 intermediate carbonaceous species of the Pt-Vulcan catalysts is better than commercial one, being them good candidates for PEMFC or DMFC.

\section{Acknowledgements}

Financial supports from the MICINN (MAT2011-24988), People ${ }_{50}$ Program (CIG-Marie Curie Actions, REA grant agreement $n^{\circ}$ 321642), "Juan de la Cierva" postdoctoral Fellowship, European Commission through the FP7 funded project ZEOCELL (no: 209481), the Government of Aragon and the European Social Fund are gratefully acknowledged.

\section{${ }_{55}$ References}

1. D. Thompsett, in Proton exchange membrane fuel cells : materials properties and performance, eds. D. P. Wilkinson, J. Zhang, R. Hui, J. Fergus and X. Li, CRC Press/Taylor \& Francis, Boca Raton, FL, 2010.

${ }_{60}$ 2.http//energy.gov/sites/prod/files/2014/12/f19/fcto_myrdd_fuel_cells.pdf .Accessed on July 2015.

3. https://setis.ec.europa.eu/system/files/Scientific_Assessment_Fuel _Cells_Hydrogen.pdf. Accessed on July 2015.

4. Z. Peng and H. Yang, Nano Today, 2009, 4, 143-164.

655 . J. L. Hueso, V. Sebastian, A. Mayoral, L. Uson, M. Arruebo and J. Santamaria, RSC Advances, 2013, 3, 10427-10433.

6. M. I. R. Borup, J. Davey, D. Wood, F. Garzon, J. Tafoya, J. Xie, S. Pacheco, DOE Hydrogen Program, FY, Progress Report, 2004.

7. P. J. Ferreira, G. J. la O', Y. Shao-Horn, D. Morgan, R. Makharia, S.

70 Kocha and H. A. Gasteiger, J. Electrochem. Soc., 2005, 152, A2256A2271.

8. L. M. Roen, C. H. Paik and T. D. Jarvic, Electrochem. Solid. St., 2004, 7, A19-A22.

9. P. L. Kuo, W. F. Chen and C. Y. Lin, J.Power Sources, 2009, 194, $75 \quad 234-242$.

10. S. Murugesan, K. Myers and V. Subramanian, Appl. Catal. BEnviron., 2011, 103, 266-274.

11. A. Eguizabal, M. A. Urbiztondo and M. P. Pina, Catal. Today., 2015, 241, 114-124.

80 12. D. Sebastian, J. C. Calderon, J. A. Gonzalez-Exposito, E. Pastor, M. V. Martinez-Huerta, I. Suelves, R. Moliner and M. J. Lazaro, Int. J. Hydrogen. Energ., 2010, 35, 9934-9942.

13. J. L. Smith, R. G. Herman, C. R. Terenna, M. R. Galler and K. Klier, J. Phys. Chem. A, 2004, 108, 39-46.

85 14. A. V. Gromov, N. Gray, P. A. Szilagyi and E. E. B. Campbell, $J$ Mater. Chem., 2012, 22, 21242-21248.

15. Y. K. Gao, F. Traeger, O. Shekhah, H. Idriss and C. Woll, J. Colloid. Interf. Sci., 2009, 338, 16-21.

16. F. Sen and G. Gokagac, J Phys Chem C, 2007, 111, 5715-5720.

90 17. G. Wu, Y. S. Chen and B. Q. Xu, Electrochem. Commun., 2005, 7, 1237-1243.

18. R-X. Wang, Y-J.Fan, L. Wang, L-N Wu and S-N. Sun, J. Power Sources, 2015, 287, 341-348.

19. J. Prabhuram, T. S. Zhao, C. W. Wong and J. W. Guo, J. Power 95 Sources, 2004, 134, 1-6. 
20. S. I. Stevanovic, D. V. Tripkovic, V. V. Panic, A. B. Dekanski and V. M. Jovanovic, RSC Advances, 2014, 4, 3051-3059..

21. I. Ávila-García, C. Ramírez, J. M. Hallen López and E. M. Arce Estrada, J. Alloy. Compd, 2010, 495, 462-465.

5 22. Z. Bai, L. Yang, J. Zhang, L. Li, C. Hu, J. Lv and Y. Guo, J.Power Sources, 2010, 195, 2653-2658.

23. D. Zhao, Y-H. Wang, B. Yan, and B-Q. Xu. J. Phys. Chem. C, 2009, 113, 1242-1250. 